\title{
Commercially available rigid gas-permeable contact lens for protecting the cornea from drying during vitrectomy with a wide viewing system
}

\author{
This article was published in the following Dove Press journal: \\ Clinical Ophthalmology \\ 13 August 2012 \\ Number of times this article has been viewed
}

\section{Motohiro Kamei \\ Nagakazu Matsumura \\ Hirokazu Sakaguchi \\ Yusuke Oshima \\ Yasushi Ikuno \\ Kohji Nishida}

Department of Ophthalmology, Osaka University Graduate School of Medicine, Osaka, Japan
Correspondence: Motohiro Kamei Department of Ophthalmology, Osaka University Graduate School of Medicine, 2-2 Yamadaoka, \#E7 Suita,

Osaka 565-087I, Japan

Email mkamei@ophthal.med.osaka-u.ac.jp
Purpose: We evaluated the usefulness of commercially available materials for protecting the cornea from drying during vitrectomy with a wide-angle viewing system.

Methods: Three vitreoretinal surgeons evaluated fundus visibility during vitrectomy on the images of five study materials used on the cornea: balanced saline solution, a viscoelastic agent, a vitrectomy contact lens, a rigid gas permeable (RGP) contact lens, and a soft contact lens. Fundus visibility was graded using the following scale: grade 1, unclear; grade 2, slightly unclear; grade 3, clear; and grade 4, very clear.

Results: Respective scores for each material used during core vitrectomy and membrane removal were: balanced saline solution, 3.3/3.3; viscoelastic agent, 3.8/3.3; vitrectomy contact lens, 3.3/4.0; RGP contact lens, 4.0/3.9; soft contact lens, 1.1/not applicable.

Conclusion: The RGP contact lens provided visibility similar to or clearer than that obtained with other methods. Because of its ease of use and lower cost, use of the RGP contact lens is ideal during vitrectomy performed with a wide-angle viewing system.

Keywords: contact lens, RGP, viscoelastic agent, vitrectomy, wide viewing system

\section{Introduction}

The varieties of wide-angle viewing systems have increased with the development of microincision vitreous surgery. The wide-angle viewing system easily provides clear fundus images from the posterior pole to near the ora serrata without the need to perform scleral indentation during vitrectomy.

Wide-angle viewing systems are either contact ${ }^{1}$ or noncontact $^{2-5}$ systems and each has advantages and disadvantages. Popular noncontact wide-angle viewing systems include the $\mathrm{BIOM}^{3}$ (Oculus, Wetzlar, Germany), OFFISS ${ }^{4}$ (Topcon Medical Systems, Oakland, NJ), Resight (Carl Zeiss Meditec AG, Jena, Germany), and Landers ${ }^{5}$ (Ocular Instruments, Belluvue, WA).

When using noncontact systems, the cornea dries out easily. Drying interferes with visibility and results in corneal erosion. Currently used techniques to avoid drying include irrigation with balanced saline solution (BSS) and application of viscoelastic agents on the cornea. Each method has limitations. BSS irrigation requires assistance to maintain the even wetness of the corneal surface, which requires frequent irrigation. When applying viscoelastic agents, the cornea should be coated evenly, and mild corneal edema sometimes is induced after approximately 20 to 40 minutes; in some cases, insurance does not cover this 
cost. A prototype of specific vitrectomy lens has recently been developed but is not yet commercially available, while currently available disposable and reusable contact vitrectomy lenses are not appropriate for use with wide viewing systems because the vitrectomy lens limits the fundus-viewing field.

Therefore, we used a commercially available rigid gas permeable (RGP) contact lens and soft contact lenses to protect the cornea from drying during vitrectomy with a wide-angle viewing system and evaluated their usefulness compared with currently available techniques.

\section{Materials and methods}

We performed phacoemulsification, intraocular lens implantation, and 25-gauge vitrectomy with a wide-angle viewing system $\left(\right.$ Resight $^{\mathbb{B}}$; Carl Zeiss Meditec AG) in three cases with epiretinal membranes. Surgical procedures were recorded using a digital video camera attached to a surgical microscope (supplementary video). To protect the cornea from drying during vitrectomy with a wide-angle viewing system, we used five materials on the cornea: BSS (BSS Plus ${ }^{\circledR}$; Alcon Japan Corp, Tokyo, Japan), a viscoelastic agent (Viscoat ${ }^{\circledR}$; Alcon Japan Corp), a vitrectomy contact lens (HHV type ${ }^{\circledR}$; HOYA, Tokyo, Japan), an RGP contact lens (HOYA HARD $\mathrm{EX}^{\circledR}$; HOYA), and a soft contact lens (Acuvue ${ }^{\circledR}$; Johnson and Johnson, Tokyo, Japan) (Figure 1). We evaluated five materials in the following order to avoid loss of time when changing materials: BSS, Viscoat, vitrectomy lens, RGP lens, and soft lens. The vitrectomy contact lens and the RGP contact lens were sterilized using low-temperature $\left(55^{\circ} \mathrm{C}\right.$ for 210 minutes) gas sterilization. The institutional review board of our institution approved this study.

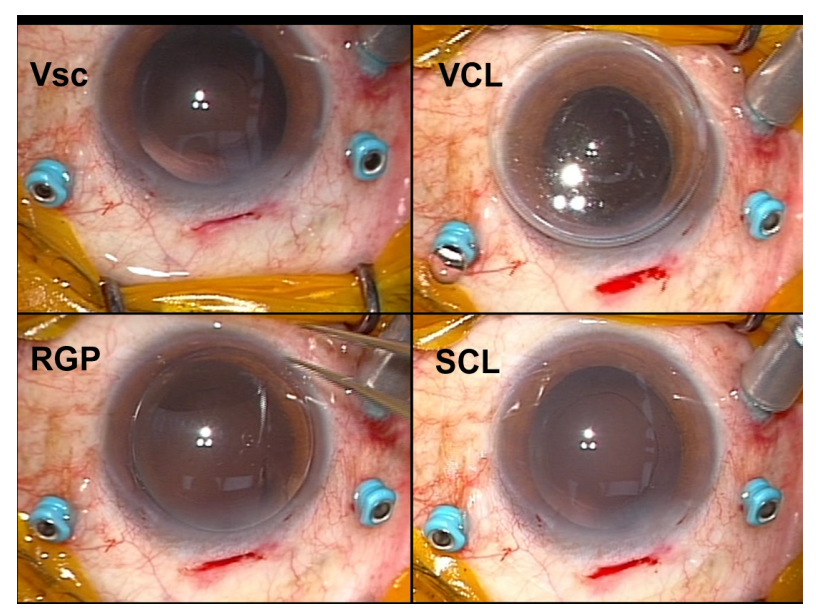

Figure I External appearance of each material in case I.

Abbreviations: RGP, rigid gas permeable contact lens; SCL, soft contact lens; $\mathrm{VCL}$, vitrectomy contact lens; Vsc, viscoelastic agent.
We evaluated fundus images obtained after the crystalline lens was removed by phacoemulsification. We fixed the magnification of the microscope and the distance between the objective lens of the wide-angle viewing system and the corneal surface. Three vitreoretinal surgeons (MK, HS, NM) graded fundus visibility on the images as follows: grade 1, unclear; grade 2, slightly unclear; grade 3, clear; and grade 4, very clear. The surgeon (MK) who performed the surgery evaluated intraoperative visibility, and the other two surgeons evaluated digital video records postoperatively in a masked fashion. Assessments were performed at the time of core vitrectomy with a 128-degree objective lens and epiretinal membrane removal and inner limiting membrane (ILM) peeling with a 60-degree objective lens.

\section{Results}

The scores for each material at the time of core vitrectomy were as follows: BSS, 3; 3-4 (median; range [min-max]); viscoelastic agent, 4; 3-4; vitrectomy contact lens, 3; 3-4; RGP contact lens, 4; 4-4; and soft contact lens, 1; 1-2 (Table 1). Figure 2 shows representative fundus images through each material. The BSS provided clear visibility, but visibility decreased after 30 to 60 seconds and frequent irrigations were required. BSS drops adhered to the objective lens of the wide-angle viewing system several times, which required that the objective lens be wiped. Visibility with the viscoelastic agent was good. The vitrectomy contact lens provided clear visibility but limited the viewing angle; the tip of the infusion cannula could not be observed without tilting the eye under the vitrectomy contact lens, but the tip was visible under the RGP contact lens. The RGP contact lens was

Table I Grades* for each material during core vitrectomy and membrane removal

\begin{tabular}{|c|c|c|c|c|}
\hline Case & $\mathbf{I}$ & 2 & 3 & Average \pm SD \\
\hline \multicolumn{5}{|c|}{ Core vitrectomy } \\
\hline BSS & $3 / 3 / 3$ & $3 / 4 / 3$ & $3 / 4 / 4$ & $3.3 \pm 0.3$ \\
\hline Vsc & $4 / 4 / 3$ & $4 / 4 / 3$ & $4 / 4 / 4$ & $3.8 \pm 0.2$ \\
\hline VCL & $3 / 4 / 3$ & $3 / 4 / 3$ & $3 / 4 / 3$ & $3.3 \pm 0$ \\
\hline RGP & $4 / 4 / 4$ & $4 / 4 / 4$ & $4 / 4 / 4$ & $4.0 \pm 0$ \\
\hline $\mathrm{SCL}$ & $2 / 1 / 1$ & $1 / 1 / 1$ & $\mathrm{I} / \mathrm{I} / \mathrm{I}$ & $1.1 \pm 0.2$ \\
\hline \multicolumn{5}{|c|}{ Membrane removal } \\
\hline BSS & $3 / 4 / 3$ & $4 / 3 / 3$ & $4 / 3 / 3$ & $3.3 \pm 0$ \\
\hline Vsc & $3 / 3 / 3$ & $4 / 4 / 3$ & $3 / 4 / 3$ & $3.3 \pm 0.3$ \\
\hline VCL & $4 / 4 / 4$ & $4 / 4 / 4$ & $4 / 4 / 4$ & $4.0 \pm 0$ \\
\hline RGP & $4 / 4 / 4$ & $4 / 4 / 4$ & $4 / 4 / 3$ & $3.9 \pm 0.2$ \\
\hline
\end{tabular}

Notes: *Grades: I, unclear; 2, slightly unclear; 3, clear; 4, very clear. Abbreviations: BSS, balanced saline solution; SCL, soft contact lens; SD, standard deviation; RGP, rigid gas permeable contact lens; VCL, vitrectomy contact lens; Vsc, viscoelastic agent. 


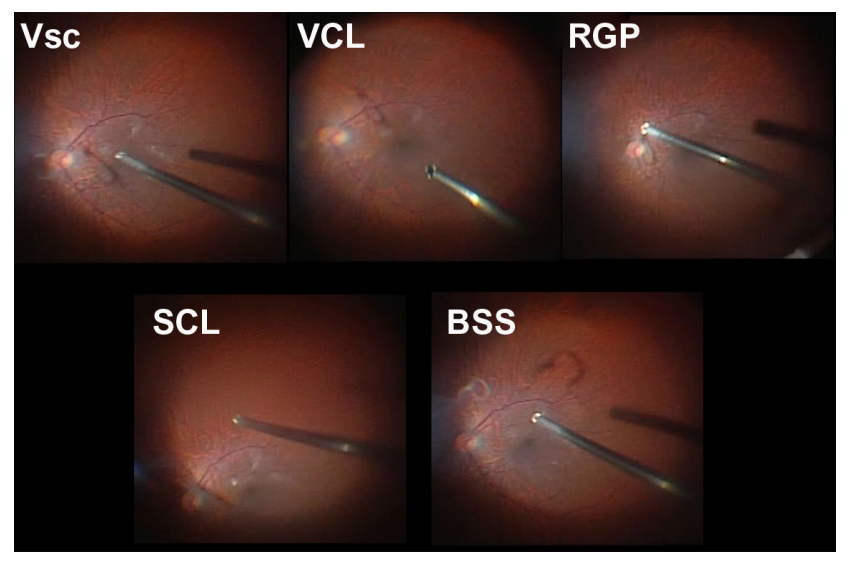

Figure 2 Representative fundus images through each material during core vitrectomy with a 128-degree objective lens.

Abbreviations: BSS, balanced saline solution; RGP, rigid gas permeable contact lens; $\mathrm{SCL}$, soft contact lens; VCL, vitrectomy contact lens; Vsc, viscoelastic contact lens.

stable on the cornea and provided stable and clear visibility. The soft contact lens curled within several minutes, resulting in blurred visibility. Exchange of the materials themselves did not affect the results because the length of the application of each material was less than a few minutes.

Scores for each material at the time of membrane removal were as follows: BSS, 3; 3-4 (median; range [min-max]); viscoelastic agent, 3; 3-4; vitrectomy contact lens, 4; 4-4, and RGP contact lens, 4; 3-4 (Table 1). Figure 3 shows representative fundus images through each material. The soft contact lens was not used during membrane removal because it curled on the cornea within several minutes and had become useless by the time of core vitrectomy. BSS and

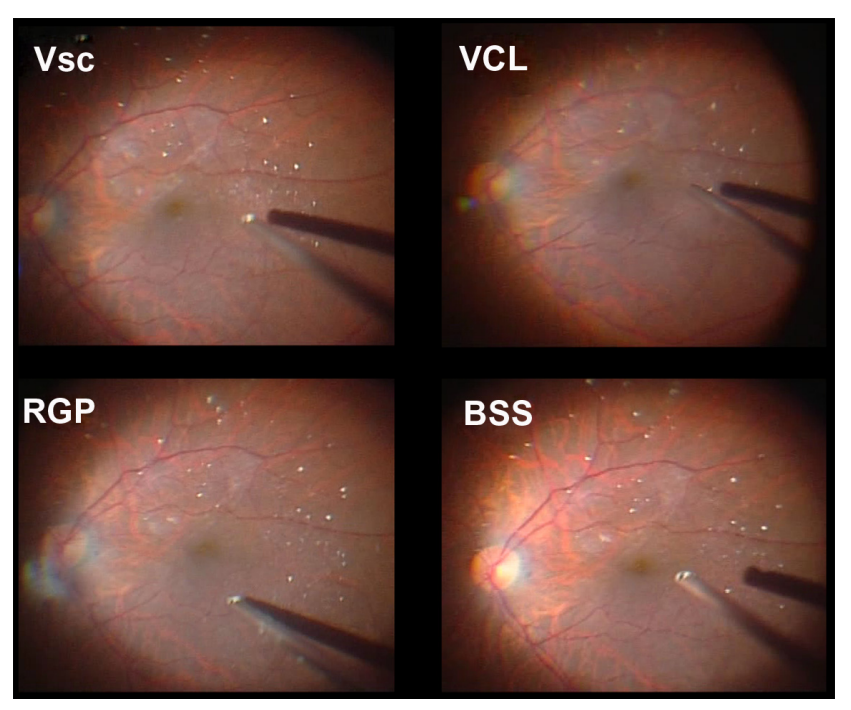

Figure 3 Representative fundus images through each material at the time of membrane peeling with 60-degree objective lens.

Abbreviations: BSS, balanced saline solution; RGP, rigid gas permeable contact lens; VCL, vitrectomy contact lens; Vsc, viscoelastic contact lens. the viscoelastic agent provided clear visibility but not as clear as the vitrectomy contact lens or the RGP contact lens. ILM peeling was difficult with BSS and the viscoelastic agent under the 60-degree objective lens of the wide-angle viewing system. With the vitrectomy contact lens and the RGP contact lens under the wide-angle viewing system, granules of triamcinolone were clearly visible and ILM peeling was performed easily.

\section{Discussion}

We examined the use of disposable soft contact lenses intraoperatively because soft contact lenses are generally used to treat corneal disorders such as postoperative corneal erosion, ${ }^{6}$ and sterile, disposable soft contact lenses are ready to use. In the current study, however, soft contact lenses did not protect the corneal surface from drying during surgery using a wide-angle viewing system. The soft contact lenses curled on the cornea and dried within several minutes, disrupting visibility. We used another type of soft contact lens (data not shown) that was slightly thicker and harder, and failed to obtain fine visibility because of the minute irregularities (waving) of the soft contact lens surface.

Interestingly, we found that the RGP contact lens provided visibility that was as clear as that obtained using BSS, viscoelastic agent, and a vitrectomy contact lens. Because of its easy handling and lower cost, an RGP contact lens appears to be ideal.

BSS also provided clear visibility at a low cost, but requires irrigation every 30 to 60 seconds. In cases in which the eye undergoing surgery is opposite to the assistant's position, it takes effort and proficiency to evenly irrigate the cornea without splashing BSS onto the objective lens. The distance between the objective lens and the corneal surface is less than $1 \mathrm{~cm}$, the operating field is dark with low room light, and microscopic illumination is not used during vitrectomy. These conditions often resulted in decreased visibility with patchy drying and interruptions of the surgery due to splashing of the BSS.

We used Viscoat, which contains sodium chondroitin sulfate and sodium hyaluronate, as the viscoelastic agent. It is more viscous and remains on the corneal surface longer than other viscoelastic agents that contain only sodium hyaluronate, which requires repeated applications. Viscoat provided clear visibility during core vitrectomy but visibility was not sufficient for fine procedures such as ILM peeling. We speculated that inability to clearly visualize fine procedures may be due to irregularities of the surface and thickness of the Viscoat. 
Vitreous surgeons are familiar with vitrectomy contact lenses because they have been used since the vitrectomy procedure was introduced. Vitrectomy contact lenses are available in all operating rooms, and disposable vitrectomy contact lenses have recently have become available. Since currently available disposable and reusable vitrectomy contact lenses are not appropriate for use with wide viewing systems, we evaluated a prototype of specific vitrectomy lens which is composed of quartz. The vitrectomy contact lenses provided sufficiently clear vision for fine procedures, but the viewing angle was limited, while BSS, viscoelastic agent, and an RGP contact lens were better for peripheral shaving. Another prototype vitrectomy contact lens has the advantage of a wider fundus view. ${ }^{7}$ Disadvantages of the vitrectomy contact lenses included poor centering on the cornea because of its weight (therefore, it required suturing a lens ring to stabilize the lens position) and higher cost than the other materials.

We used a commercially available RGP contact lens that was not sufficiently sterilized for intraocular surgery and required ethylene oxide gas sterilization. The RGP contact lens did not deform with low-temperature ethylene oxide gas sterilization (ethylene oxide gas at $55^{\circ} \mathrm{C}$ for 3.5 hours and aeration at $55^{\circ} \mathrm{C}$ for 8 hours). The labor, time, and cost of the sterilization are limitations of the RGP contact lens use. Manufacturers must provide a sterilized, disposable, low-cost RGP contact lens.

We used a -3-diopter RGP contact lens. We are now conducting a study to compare RGP contact lens powers.
Preliminary results indicate that different lens powers do not make a significant difference, although lenses with plus diopters limit the viewing angle with higher magnification.

This pilot study was limited because of the small number of cases; we were unable to examine statistical significance. Therefore, we are conducting a multicenter prospective study to include more cases and surgeons.

In conclusion, we recommend using an RGP contact lens to protect the cornea from drying during vitrectomy with a wide-angle viewing system.

\section{Disclosure}

The authors report no conflicts of interest in this work.

\section{References}

1. Nakata K, Ohji M, Ikuno Y, et al. Wide-angle viewing lens for vitrectomy. Am J Ophthalmol. 2004;137:760-762.

2. Bovey EH, Gonvers M. A new device for noncontact wide-angle viewing of the fundus during vitrectomy. Arch Ophthalmol. 1995;113: $1572-1573$.

3. Spitznas M. A binocular indirect ophthalmomicroscope (BIOM) for noncontact wide-angle vitreous surgery. Graefes Arch Clin Exp Ophthalmol. 1987;225:13-15.

4. Horiguchi M, Kojima Y, Shimada Y. New system for fiberoptic-free bimanual vitreous surgery. Arch Ophthalmol. 2002;120:491-494.

5. Landers MB, Peyman GA, Wessels IF, Whalen P, Morales V. A new, non-contact wide field viewing system for vitreous surgery. Am J Ophthalmol. 2003;136:199-201.

6. Arentsen JJ, Tasman W. Using a bandage contact lens to prevent recurrent corneal erosion during photocoagulation in patients with diabetes. $\mathrm{Am} \mathrm{J}$ Ophthalmol. 1981;92:714-716.

7. Ohji M, Tada E, Futamura H. Combining a contact lens and wide-angle viewing system for a wider fundus view. Retina. 2011;31:1958-1960.
Clinical Ophthalmology

\section{Publish your work in this journal}

Clinical Ophthalmology is an international, peer-reviewed journal covering all subspecialties within ophthalmology. Key topics include: Optometry; Visual science; Pharmacology and drug therapy in eye diseases; Basic Sciences; Primary and Secondary eye care; Patient Safety and Quality of Care Improvements. This journal is indexed on Submit your manuscript here: http://www.dovepress.com/clinical-ophthalmology-journal

\section{Dovepress}

PubMed Central and CAS, and is the official journal of The Society of Clinical Ophthalmology (SCO). The manuscript management system is completely online and includes a very quick and fair peer-review system, which is all easy to use. Visit http://www.dovepress.com/ testimonials.php to read real quotes from published authors. 